\title{
Bioequivalence of Branded and Generic Oxaliplatin: From Preclinical Assessment to Clinical Incidence of Hypersensitivity Reactions
}

\author{
MARCO TAMPELLINI ${ }^{1}$, SIMONE BENEDETTO ${ }^{1}$, ELENA RUBATTO $^{1}$, CHIARA BARATELLI $^{1}$, \\ FEDERICA DI SCIPIO ${ }^{2}$, ELISA PIRRO ${ }^{2}$, MARIA PIA BRIZZI ${ }^{1}$, CRISTINA SONETTO $^{1}$, \\ MASSIMO DI MAIO ${ }^{1}$, GIOVANNI NICOLAO BERTA ${ }^{2}$ and GIORGIO VITTORIO SCAGLIOTTI ${ }^{1}$ \\ ${ }^{1}$ Division of Medical Oncology and ${ }^{2}$ Division of Pharmacology, University of Torino, \\ Department of Oncology, S. Luigi Hospital, Turin, Italy
}

\begin{abstract}
Background: Generic anticancer drugs represent an opportunity in terms of cost savings but there are some concerns about their tolerability. The safety profiles of generic versus branded oxaliplatin formulations have never been studied in detail. Patients and Methods: We tested in vitro concentrations, stability and efficacy of branded versus generic oxaliplatin formulations, then we retrospectively collected data about hypersensitivity reactions (HSR) of 427 colorectal cancer patients treated with oxaliplatin-based regimens. Results: No significant difference in oxaliplatin concentration or time-dependent antiproliferative activity between branded and generic oxaliplatin was detected. The incidence of HSR was $12.1 \%$ (33/273 patients) in those treated with branded and $9.8 \%$ (15/154 patients) in those treated with generic oxaliplatin $(p=0.46)$. The occurrence of grade III-IV HSRs and severe HSRs leading to oxaliplatin discontinuation were comparable. Conclusion: No difference between generic and branded formulations of oxaliplatin were demonstrated in preclinical nor in clinical settings. Generic oxaliplatin can be considered a safe alternative to branded formulation.
\end{abstract}

Chemotherapy for colorectal cancer (CRC) has radically changed in the past decades since new agents have been added to the regimen of 5-fluorouracil (5-FU)/leucovorin (LV), which still remains the backbone of all systemic treatments. In the late 1990 s, the incorporation of irinotecan

Correspondence to: Cristina Sonetto, MD, Department of Oncology, University of Torino, AOU San Luigi di Orbassano, Regione Gonzole 10, 10043 Orbassano, Italy. Tel: +390119026526, Fax: +390119026992, e-mail: cristina.sonetto@gmail.com

Key Words: Metastatic colorectal cancer, generic oxaliplatin, hypersenstitivity reactions, bioequivalence. and oxaliplatin into the treatment armamentarium improved patient outcomes, and, consequently, irinotecan plus 5FU/LV (FOLFIRI) and oxaliplatin plus 5FU/LV (FOLFOX) regimens became the standard of care (SOC) in advanced disease. Furthermore, oxaliplatin was tested in the adjuvant setting, and it is now SOC because of a demonstrated reduction in relapse rate and an improvement in overall survival when combined with a fluoropyrimidine compared to fluoropyrimidine-alone in patients with stage III CRC and, to a lesser extent, in those with stage II. Thus, oxaliplatin has become one of the most used drugs in patients with CRC.

The cost of anticancer therapies is rapidly growing, and any strategy leading to cost savings is always welcome (1). Generic drugs (commonly called generics) represent a relevant, potential opportunity in terms of cost savings. WHO defines a generic as "a pharmaceutical product, usually intended to be interchangeable with an innovator product, that is manufactured without a license from the innovator company and marketed after the expiry date of the patent or other exclusive rights" (2). Generics are usually sold for significantly lower prices than their branded equivalents and data extracted from the Pharmacy Registry of our Institution revealed that on December 18th 2008, the cost of one vial of oxaliplatin (100 mg) was $€ 252.9$ for Eloxatin $^{\mathrm{TM}}$ (Sanofi Aventis ${ }^{\mathrm{TM}}$ ) and $€ 38.2$ for generic oxaliplatin (Ebewe ${ }^{\mathrm{TM}}$ ). Several reasons account for the lower price of generic drugs, including increased competition among producers when drugs are no longer protected by patents; generic manufacturers do not incur costs related to drug discovery; generic manufacturers are not required to test the safety and efficacy of the drugs through clinical trials, since those steps have already been conducted by the branded name company.

The administration of oxaliplatin (both branded and generic) is associated with hypersensitivity reactions (HSR) in about $10 \%$ of patients (3). These HSRs are mainly 
characterized by an early onset during drug infusion (even though some delayed events have been reported), and different symptoms, predominantly cutaneous side-effects. In some cases, more severe and life-threatening symptoms occur. The prophylactic administration of premedication including steroids and antihistamines allows oxaliplatin re-challenge and continuation of treatment in less than one-third of patients ( 3 , 4). As a whole, about $7 \%$ of all patients receiving an oxaliplatin-containing regimen discontinue therapy, with a potentially detrimental effect in terms of tumor curability (adjuvant setting) or survival (advanced setting).

With the aim of reporting the HSR incidence and overall toxicity rates before and after the introduction of generic oxaliplatin, we (i) tested the concentrations, stability and efficacy of branded formulation versus generics ones in vitro, and (ii) retrospectively collected data of all patients with CRC followed-up at our Institution, comparing the data in patients treated with the branded drug with those of patients treated with generic formulations.

\section{Patients and Methods}

Chromatographic analyses. In order to determine the actual concentration of the considered oxaliplatin formulations (branded and generic), we reconstituted the samples according to the manufacturer's indications (if necessary). They were randomly chosen from among the batches used for patients at our Institution. At the time of preclinical assessment, the branded oxaliplatin formulation was Eloxatin ${ }^{\mathrm{TM}}$ from Sanofi Aventis and two generic formulations were Ebewe ${ }^{\mathrm{TM}}$ and $\mathrm{Teva}^{\mathrm{TM}}$.

Samples were prepared at a concentration of $5 \mathrm{mg} / \mathrm{ml}$ of oxaliplatin and each sample was supplemented with flavone (250 $\mu \mathrm{g} / \mathrm{ml}$ in methanol) which acted as an internal standard. The chromatographic analysis was performed with a LaChrom highperformance liquid chromatography (HPLC) apparatus equipped with UV spectrophotometer (VWR International, Milan, Italy). Following an injection of $10 \mu \mathrm{l}$ of the sample, separation was achieved with a C18 reversed-phase column (LiChroCART ${ }^{\mathrm{TM}}$ 250$4 \mathrm{~mm}$ LiChrospher ${ }^{\mathrm{TM}} 100 \mathrm{RP}-18,5 \mu \mathrm{m}$; VWR International)

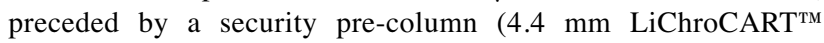
LiChrospher $^{\mathrm{TM}} 100 \mathrm{RP}-18,5 \mathrm{~m}$; VWR International). The isocratic mobile phase consisted of water and acetonitrile $(30: 70, \mathrm{v} / \mathrm{v})$. Analyses were carried out at a constant flow rate of $1 \mathrm{ml} / \mathrm{min}$ at $30^{\circ} \mathrm{C}$ and the elute was monitored at $255 \mathrm{~nm}$ for $6 \mathrm{~min}(5)$.

In order to test the stability of formulations, we analyzed such samples prepared as above after storage for 1,3 , and 7 days at room temperature, $4^{\circ} \mathrm{C}$, and $37^{\circ} \mathrm{C}$.

Cytotoxicity assay. The CACO-2 cell line (Sigma Aldrich, Milano, Italy) derived from human colorectal adenocarcinoma (6) was cultured in $75 \mathrm{~cm}^{2}$ flasks (TPP AG, Trasadingen, Switzerland) in DMEM+GlutaMAX ${ }^{\mathrm{TM}}$ (Life Technologies Europe BV, Monza MB, Italy) supplemented with $10 \%$ fetal bovine serum, $100 \mathrm{U} / \mathrm{ml}$ penicillin G, $40 \mu \mathrm{g} / \mathrm{ml}$ gentamicin sulfate and $2.5 \mu \mathrm{g} / \mathrm{ml}$ amphotericin $\mathrm{B}$ at $37^{\circ} \mathrm{C}$ in a humidified atmosphere with $5 \% \mathrm{CO}_{2}$ (unless otherwise specified, materials were from Sigma-Aldrich, Milan, Italy). Cytotoxicity assays were performed as previously described (7). Briefly, cells were seeded at a density of $2 \times 10^{3}$ per well in a 96-well plate (TPP AG). After 1 day, the medium was replaced and supplemented with different concentrations (ranging from 5 to $50 \mu \mathrm{M}$ ) of tested oxaliplatin formulations. After 48 and $72 \mathrm{~h}$ of incubation, cell viability was evaluated by 3-(4,5dimethylthiazol-2-yl)-2,5-diphenyl-tetrazolium bromide (MTT) assay and the results expressed as the average percentage of absorbance of treated versus control (culture medium alone) cells. Reported results are the mean of three independent experiments.

Patients. Clinical data and outcomes of all patients with CRC treated at our Institution were retrieved from our institutional database with data prospectively collected since 1993. Data between January 1st,1994 and June 30th, 2014 from patients who received first-line or adjuvant treatment with oxaliplatin-containing regimens were then extracted and entered into a new database specifically designed for the present study. The data extracted included patient demographics, primary tumor characteristics with stage and grading, disease-free interval, site of metastasis (if any), chemotherapy history, date of first progression and date of death or last follow-up visit.

Patients were predominantly treated with the FOLFOX, XELOX or the chronomodulated (CHRONO) regimens. FOLFOX consisted of $100 \mathrm{mg} / \mathrm{m}^{2}$ oxaliplatin on day 1 , followed by 2 hour-infusion of $300 \mathrm{mg} / \mathrm{m}^{2} \mathrm{LV}$ and 22 hour-infusion of $1500 \mathrm{mg} / \mathrm{m}^{2} 5-\mathrm{FU}$. On day 2, patients received 2-hour infusion of $300 \mathrm{mg} / \mathrm{m}^{2} \mathrm{LV}$ and 22-hour infusion of $1500 \mathrm{mg} / \mathrm{m}^{2} 5-\mathrm{FU}$; the cycle was repeated every 14 days. XELOX regimen contained $100 \mathrm{mg} / \mathrm{m}^{2}$ oxaliplatin on day 1 and $2000 \mathrm{mg} / \mathrm{m}^{2}$ capecitabine b.i.d. for 14 consecutive days; the cycle was repeated every 21 days. The CHRONO regimen was administered by means of automatic, programmable out-patient pumps which delivered drugs during a predetermined time-frame and with a flow rate varying according to a sinusoidal curve. Patients received a 4-day course of chronomodulated infusions of 5 -FU $\left(750 \mathrm{mg} / \mathrm{m}^{2} /\right.$ day $)$ - LV $\left(300 \mathrm{mg} / \mathrm{m}^{2} /\right.$ day $)$ delivered at night (from $22: 15$ to $09: 45$ hours with a peak at 0400 hours), and oxaliplatin $\left(25 \mathrm{mg} / \mathrm{m}^{2} /\right.$ day) delivered during the day (from 10:15 to 21:45 hours with a peak at 1600 hours).

Patients were grouped into two subgroups according whether they received branded or generic oxaliplatin. Data about the type of oxaliplatin administered were extracted from the Pharmacy Log of our Institution. In general, Eloxatin ${ }^{\mathrm{TM}}$ (Sanofi-Aventis ${ }^{\mathrm{TM}}$, branded) was administered up to January 15, 2009; Ebewe ${ }^{\mathrm{TM}}$ (generic) from January 16, 2009 until December 10, 2009; Hospira ${ }^{\text {TM }}$ (generic) from December 11, 2009 until March 17, 2011; and finally Teva ${ }^{\mathrm{TM}}$ (generic) from March 18, 2011 until the end of the study. Patients receiving both branded and any generic formulation were excluded from the analysis.

Assessment of HSRs and toxicities. HSRs were defined as one of the following symptoms occurring during or immediately after oxaliplatin infusion: rash, itching, urticaria, flushing, burning, palmar erythema, edema of the face and hands, abdominal cramping, diarrhea, back pain, tachycardia, bronchospasm, hypotension or hypertension, and seizures.

Central laboratory database and clinical charts were the sources of data for hematological and non-hematological toxicities, respectively. Toxicity was recorded per cycle of therapy and graded according to version 3.0 of the Common Terminology Criteria for Adverse Events (8). The worst toxicity per patient was then considered for the statistical analyses. On the basis of the findings 
published by Giacchetti et al. (9) in which a different toxicity profile was demonstrated in patients submitted to chronotherapy versus those who received FOLFOX, the former patients were excluded from the toxicity analyses. The remaining patients, who received FOLFOX or XELOX regimen, were then grouped according whether they presented no or only mild toxicity (grade 0 and 1) versus moderate to severe toxicity (grade 2, 3 and 4).

Outcomes assessment. Treatment response was classified according to the Union for International Cancer Control criteria (10) up to 2001, and then to Response Evaluation Criteria In Solid Tumors (RECIST) (11). Complete response (CR) was defined as the complete disappearance of all clinically detectable malignant disease. As far as the UICC criteria are concerned, a partial response (PR) was defined as a decrease of $>50 \%$ in the sum of the products of the two longest perpendicular diameters of all measurable lesions, and progressive disease (PD) was defined as an increase of at least $25 \%$ in the size of measurable lesions and the development of new lesions. According to the RECIST criteria, the definition of PR and PD are a decrease by $>30 \%$ and an increase by $>20 \%$ of the sum of the longest diameters of the target lesions, respectively (11). Only the best tumor response was recorded.

For patients treated for advanced disease, progression-free survival (PFS) was estimated from the start of first-line treatment to the date of disease progression or death (whichever came first) or to the date of the last follow-up visit for censored patients. Overall survival (OS) was considered from the start of first-line treatment to the date of death, or date of the last follow-up for censored patients. The cut-off date for statistics computation was June 30th, 2015.

Statistical analyses. The calibration curves for HPLC analyses were constructed by plotting the peak-height ratios of oxaliplatin to the internal standard versus the nominal concentrations in the standard biological samples using linear regression analysis. Oxaliplatin concentrations are reported as the mean \pm standard deviation (SD). Two-way ANOVA and Bonferroni multiple comparison test (for post-ANOVA comparisons) were used to evaluate the statistical significance of the preclinical experiments.

For patients, differences between proportions were evaluated using the chi-square test with Yates correction, when appropriate. Differences between groups by non-parametric unpaired variables were validated by the Mann-Whitney $U$-test. Considering the high number of statistical inferences planned in the study $(>20)$, in order to avoid a validation due to chance and not to a real difference, we considered a value of $p<0.01$ as valid. With this cut-off, we limited the number of statistical inferences incorrectly considered valid from 1 out of 20 to 1 out of 100 . Survival curves were plotted using the Kaplan-Meier method and compared using the log-rank test. All statistical computations were performed using GraphPad Prism version 6.0c for Mac OSX (GraphPad software, La Jolla, CA, USA), SPSS for Windows Ver 22.0 (IBM Software, Segrate, MI, Italy), and STATISTICA for Windows Ver. 8.0 (Statsoft, Dell Software, Tulsa, OK, USA).

\section{Results}

Preclinical bioequivalence and stability of oxaliplatin formulations. In order to test the reported percentage of oxaliplatin in the different batches (branded and generics), we analyzed the freshly opened samples commercially available by means of HPLC. Although all three drugs were a little less concentrated than stated, there were no significant differences in oxaliplatin concentration between Eloxatin $^{\mathrm{TM}}$ and the generic formulations or among all generic drugs tested (Figure 1A). Moreover, in order to assess the stability of formulations following reconstitution, we examined the samples after storage for 1,3 , and 7 days at room temperature, $4^{\circ} \mathrm{C}$, and $37^{\circ} \mathrm{C}$. Our HPLC data demonstrated that different storage conditions did not significantly affect drug stability compared to freshly opened formulations for all considered samples (Figure 1B).

The in vitro efficacy of the considered formulations on colorectal adenocarcinoma cell line was examined by growing CACO-2 cells with an increasing amount of Eloxatin $^{\mathrm{TM}}$, Ebewe ${ }^{\mathrm{TM}}$ and $\mathrm{Teva}^{\mathrm{TM}}(5,15,50 \mu \mathrm{M})$ and measuring the viability by a colorimetric assay at 48 and 72 h. All three formulations exhibited dose- and timedependent antiproliferative activity against CACO-2 cells, but for each concentration and time there were no significant differences between Eloxatin ${ }^{\mathrm{TM}}$ and the generic formulations (Figure 1C).

Patients. A total of 427 patients were included in the analysis and clinical and treatment characteristics are summarized in Table I. The majority were males $(61.1 \%)$, and presented with synchronous metastases $(63.0 \%)$. Main patient characteristics in the two oxaliplatin-treated groups were similar, with the following exceptions: patients in the generic-treated group were older (median age $=67.7 \mathrm{vs} .64 .9$ years, $p<0.002$ ), and were treated predominantly with the FOLFOX or the XELOX regimen as compared to the chronomodulated therapy $(91.6 \% v s .35 .5 \%, p<0.001)$.

A total of 4100 cycles were entered into the database. Their distribution according to type of chemotherapy and oxaliplatin formulation is summarized in Table I. Patients received a median of 11 cycles of oxaliplatin (range=1-16): 12 (1-14) of FOLFOX, 12 (1-16) of chronotherapy, and 8 (112) of XELOX. Patients in the brand-treated group received the chronotherapy regimen more frequently than did the generic-treated group (64.5\% vs. 8.4\%), whereas FOLFOX was more frequently administered in the generic-treated group $(61.7 \%$ vs. $26.7 \%)(p<0.0001)$.

Hypersensitivity reactions. A total of 48 patients $(11.3 \%)$ experienced at least one HSR (Table II). In the group treated with branded drug, 33 out of 273 patients (12.1\%) had an HSR, whereas this was the case for 15 out of 154 patients $(9.8 \%)$ in the generic-treated group. This difference was not statistically significant $(p=0.46)$. No difference in time to occurrence of HSR was demonstrated between the two groups (log-rank $p=0.57$ ) (Figure 2 ). The most frequent HSRs were palma-plantar erythema or facial rash, often associated with itching. In four cases, laryngospasm was 
A
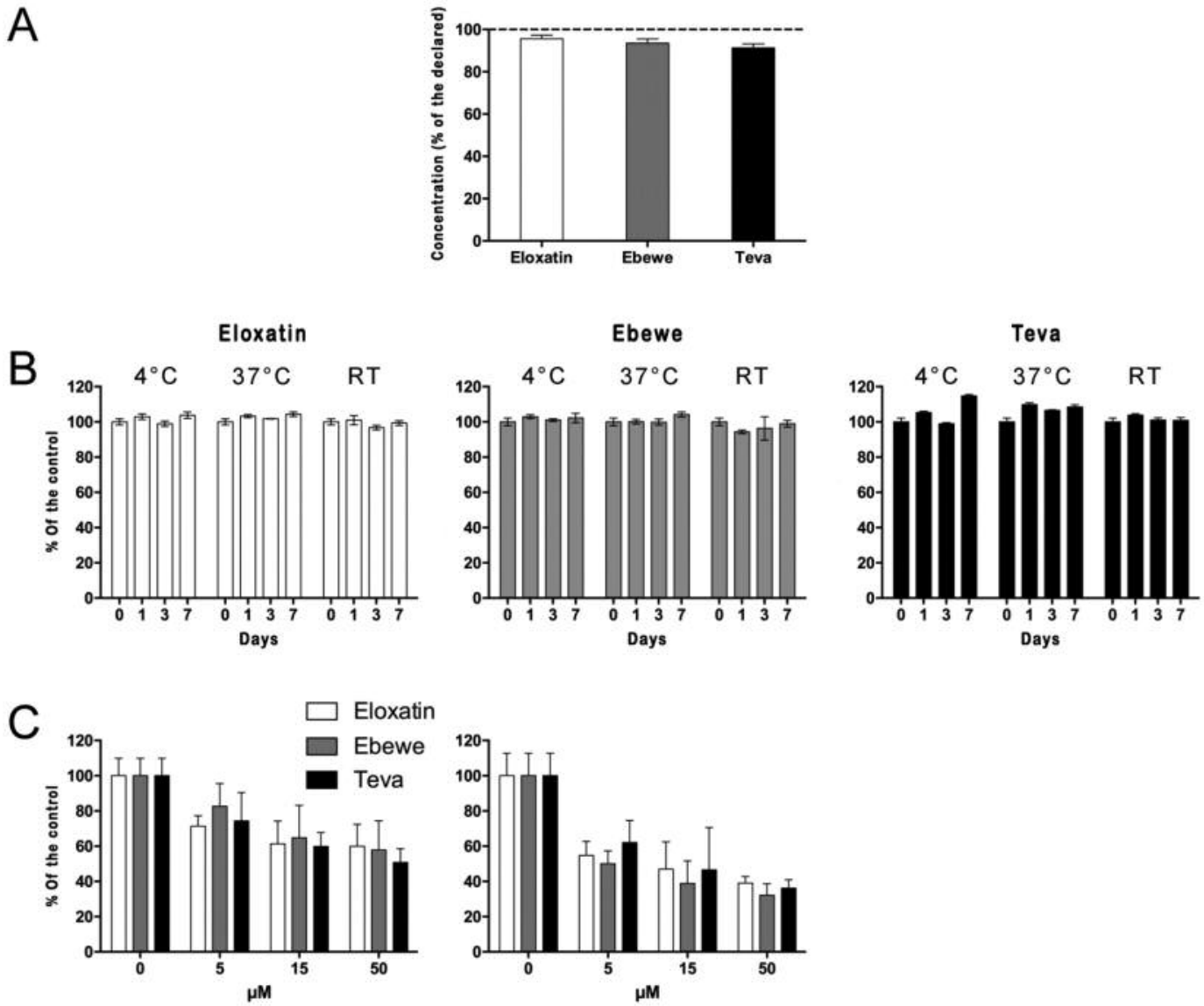

Figure 1. Preclinical bioequivalence and stability of oxaliplatin formulations: differences in oxaliplatin concentration (A), stability under different storage conditions $(B)$, and dose-dependent antiproliferative activity against CACO-2 cells 24 hours (left) and 48 hours (right) after drug exposure (C). Eloxatin ${ }^{T M}$ : Branded drug; Ebewe ${ }^{T M}$ and Teva ${ }^{T M}$ : generic drugs.

reported, without laboratory evidence of desaturation or shock. Fever was reported in one case, a few minutes after the first cycle (maximum $38.8^{\circ} \mathrm{C}$ ) and at the end of the second oxaliplatin administration, notwithstanding premedication with $1,000 \mathrm{mg}$ paracetamol per os. The oxaliplatin dose was then reduced to $50 \%$ and the patient continued chemotherapy without any subsequent additional side-effect.

Grade III-IV HSRs were recorded in 16 patients (3.7\%), $11(4.0 \%)$ in the group treated with branded drug and five $\left(3.2 \%, \chi^{2} p=0.8\right)$ in the group treated with generic drug. They mainly consisted of hypotension recovered in few minutes after drug discontinuation. The median number (range) of cycles at which the severe HSRs occurred was 4 (1-12) in the overall population, 5 (1-12) in the brand-treated group and $3(1-12)$ in the generic-treated group $(p=0.98)$. Finally, severe HSRs led to oxaliplatin discontinuation in 14 patients, nine $(3.3 \%)$ and five $(3.2 \%)$ in the brand- and generic-treated groups, respectively $(p=0.98)$.
Clinical outcomes. Chemotherapy was generally well tolerated, with grade 3 and 4 toxicities reported in fewer than $10 \%$ of the patients. The most frequent were: neutropenia in 41 patients $(9.6 \%$; 38 of grade 3 and three of grade 4) and diarrhea in 25 patients $(5.8 \% ; 21$ grade 3 and four of grade 4). Toxicities in patients treated with FOLFOX or XELOX regimens and grouped according to grade of intensity are listed in Table III. No major difference between the two groups was evident, with the only exception being leucopenia $(31.2 \%$ vs. $18.6 \%$, in brand- and generic-treated groups, respectively, $p=0.03$ ).

Considering only the 398 patients with metastatic CRC, no difference in response rate between groups was evident (43.2\% vs. $43.1 \%$, in brand- and generic-treated groups, respectively). For the whole patient cohort, PFS and OS were 13.6 and 25.4 months, respectively. A longer median PFS was demonstrated in the brand-treated group (14.4 vs. 12.4 months, log-rank $p<0.03$ ), whereas OS was not significantly different between the two groups (26.9 vs. 24.9 months, respectively, log-rank $p=0.14$ ). 


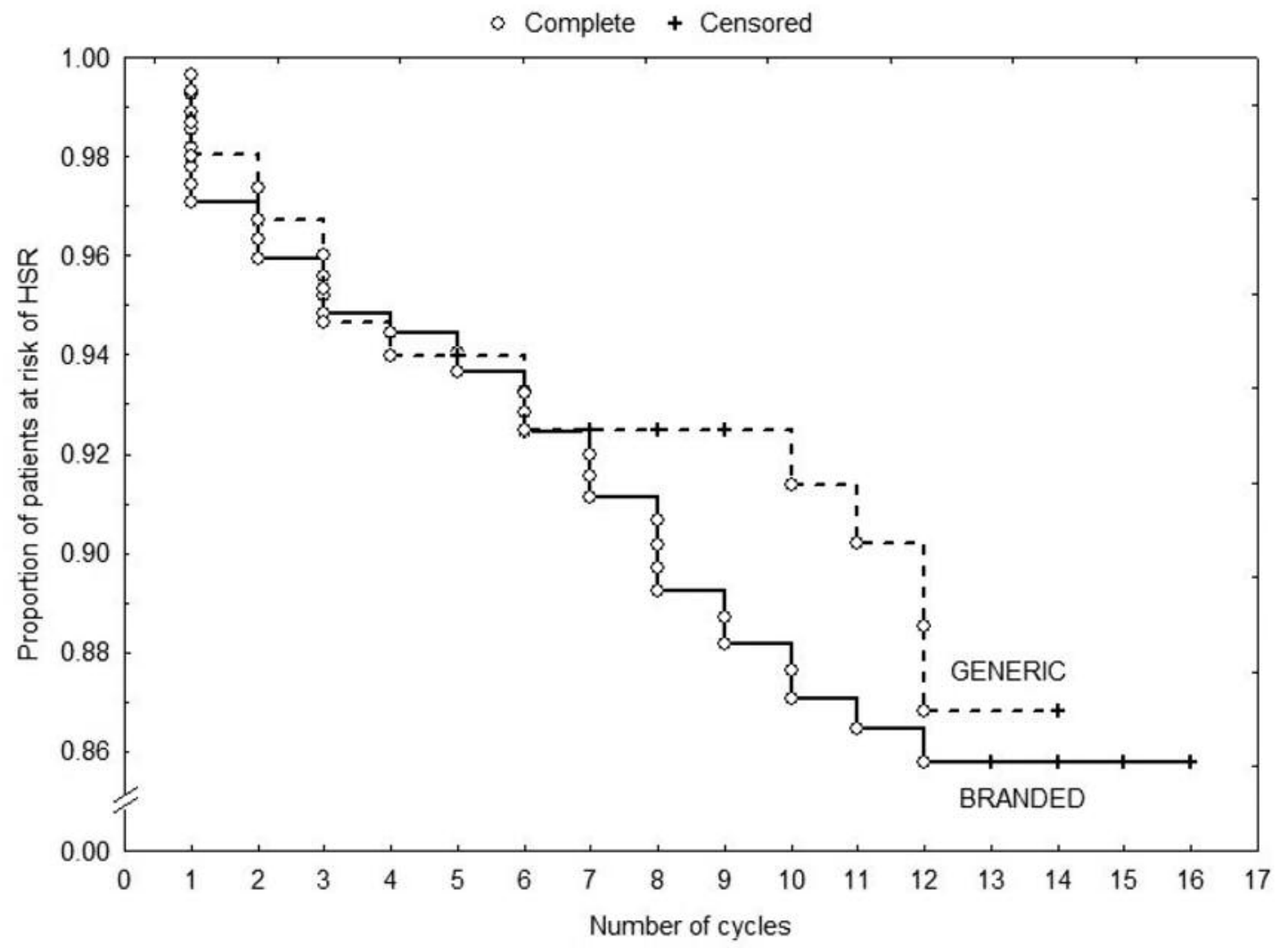

Figure 2. Time to occurrence of oxaliplatin-induced hypersensitivity reactions (HSR) for patients stratified according to drug formulation (log-rank $p=0.57$ ).

\section{Discussion}

Notwithstanding the European Medicine Agency and the other regulatory agencies guidelines guaranteeing the quality of available generic drugs, physicians might be uncomfortable with patients taking the generic equivalents instead of the branded drugs. The main concern is related to a potential low quality of the generic formulation, theoretically leading to a lesser pharmacological effect and a higher incidence of adverse events. Although the available literature is still lacking straightforward evidence and often the negative point of view of physicians is frequently tied to anecdotal cases without any solid scientific evidence, the use of different formulations of the same active agent may also increase the frequency and severity of side-effects. The former point, leads to the hypothesis that different formulations may differ in bioequivalence (mainly in terms of concentrations of the active agent). In fact, in order to define the bioequivalence of the generic product compared to the innovator, current laws require only that the $90 \%$ confidence interval of pharmacokinetic parameters (area under the curve ratio and maximal concentration ratio) are between $80 \%$ and $125 \%$. However, bioequivalence tests are generally not required if the generic agent is administered as aqueous intravenous solution containing the same active agent as the currently approved drug (12).

Secondly, there is a potential threat related to a potential increase in the incidence of the side-effects mainly related to different formulations of the same drug with different excipients. Thus, some patients who did not have any adverse event when taking a branded drug may develop allergic reactions or other adverse effects when using another formulation of the same active agent. Excipients that have been known to generate adverse effects include sulfites, saccharin, aspartame, benzyl alcohol, lactose, propylene glycol and soya lecithin (13).

Additionally, it is known that some excipients may influence drug bioavailability and a different combination of excipients may change the rate at which the drug is released, when it should be administered, and a food and liquid interaction may be observed (14). Hence, specific tests should be conducted when excipients are different, at different concentrations, or because a possible interaction with the active agent (e.g. complex formation), or otherwise affect the disposition of the drug.

For these reasons, we performed this preclinical and retrospective clinical study to improve information about 
Table I. Patient characteristics according to drug formulation.

\begin{tabular}{|c|c|c|c|}
\hline & Total & Branded drug & Generic drug \\
\hline No. of patients $(\%)$ & 427 & $273(63.9)$ & $154(36.1)$ \\
\hline Male & $261(61.1)$ & $167(61.2)$ & $94(61.0)$ \\
\hline Female & $166(38.9)$ & $106(38.8)$ & $60(39.0)$ \\
\hline Median age (range), years & $65.6(22.2-84.1)$ & $64.9(28.9-84.1)$ & $67.7(22.2-82.5)$ \\
\hline \multicolumn{4}{|l|}{ Stage at diagnosis, n (\%) } \\
\hline A & $3(0.7)$ & $3(1.1)$ & 0 \\
\hline $\mathrm{B}$ & $50(11.7)$ & $29(10.6)$ & $21(13.6)$ \\
\hline $\mathrm{C}$ & $101(23.7)$ & $67(24.6)$ & $34(22.1)$ \\
\hline $\mathrm{D}$ & $269(63.0)$ & $171(62.6)$ & $98(63.7)$ \\
\hline Unknown & $4(0.9)$ & $3(1.1)$ & $1(0.6)$ \\
\hline \multicolumn{4}{|l|}{ Chemotherapy setting, $\mathrm{n}(\%)$} \\
\hline Adjuvant & 29 & 5 & 24 \\
\hline Metastatic & 398 & 268 & 130 \\
\hline \multicolumn{4}{|l|}{ Site of metastases, $\mathrm{n}(\%)$} \\
\hline Liver & $287(67.2)$ & $188(68.9)$ & $99(64.3)$ \\
\hline Lung & $139(32.6)$ & $89(32.6) 1$ & $50(32.5)$ \\
\hline Other & $142(33.3)$ & $89(32.6)$ & $53(34.4)$ \\
\hline \multicolumn{4}{|l|}{ Treatment regimen } \\
\hline Total no. of cycles $(\%)$ & 4100 & $2665(65.0)$ & $1435(35)$ \\
\hline \multicolumn{4}{|l|}{ CHRONO } \\
\hline No. of patients (\%) & $189(44.3)$ & $176(64.5)$ & $13(8.4)$ \\
\hline Total no. of cycle administered $(\%)$ & $1910(46.6)$ & $1778(66.7)$ & $132(9.2)$ \\
\hline Median no. of cycle administered per patient (range) & $12(1-16)$ & $12(1-16)$ & $12(3-12)$ \\
\hline \multicolumn{4}{|l|}{ FOLFOX } \\
\hline No. of patients $(\%)$ & $168(39.3)$ & $73(26.7)$ & $95(61.7)$ \\
\hline Total no. of cycle administered (\%) & $1705(41.6)$ & $714(26.8)$ & $991(69.1)$ \\
\hline Median no. of cycles administered per patient (range) & $12(1-14)$ & $11(1-13)$ & $12(2-14)$ \\
\hline \multicolumn{4}{|l|}{ XELOX } \\
\hline No. of patients $(\%)$ & $70(16.4)$ & $24(8.8)$ & $46(29.9)$ \\
\hline Total no. of cycle administered (\%) & $485(11.8)$ & $173(6.5)$ & $312(21.7)$ \\
\hline Median no. of cycle administered per patient (range) & $8(1-12)$ & $8(1-12)$ & $8(2-10)$ \\
\hline \multicolumn{4}{|l|}{ Response to chemotherapy, $\mathrm{n}$ ( $\%$ of eligible patients) } \\
\hline Response & $173(43.1)$ & $110(43.1)$ & $63(43.2)$ \\
\hline Stabilization & $163(40.6)$ & $98(38.4)$ & $65(44.5)$ \\
\hline Progression & $65(16.3)$ & $47(18.5)$ & $18(12.3)$ \\
\hline Unknown (\% of entire population) & $26(6.1)$ & $18(6.6)$ & $8(5.2)$ \\
\hline
\end{tabular}

CHRONO: 5-Fluorouracil, leucovorin and oxaliplatin administered according to a chronomodulated schedule; FOLFOX: 5-fluorouracil, leucovorin and oxaliplatin: XELOX: capecitabine and oxaliplatin.

branded oxaliplatin and its generic forms. More specifically, we carried out a series of preclinical in vitro analyses to highlight the possible bioequivalence between Eloxatin ${ }^{\mathrm{TM}}$ and its generics, in terms of: a) concentration of active agent in the formulations, b) stability of the formulations under different temperatures (room temperature, $4^{\circ} \mathrm{C}$, or $37^{\circ} \mathrm{C}$ ), and c) efficacy of the formulations in vitro. Our data show that there were no significant differences between the different formulations (Eloxatin ${ }^{\mathrm{TM}}$ and the generic formulations, nor among the latter) for the considered parameters.

Therefore, after excluding bioequivalence problems, we carried out a retrospective clinical study on patients to investigate whether excipients in the different formulations were responsible for any difference in adverse reactions.
The occurrence of oxaliplatin-induced HSRs is common. In a recent review, global incidence ranged from $8.9 \%$ to $23.8 \%$ according to different series, whereas grade III-IV HSRs were present in $1.6-14.6 \%$ of patients (15). This wide range in HSR incidence might be explained by the heterogeneity of the clinical manifestations, which are variable and unpredictable, thus not always interpreted by physicians as being associated with oxaliplatin. With this limitation, although no publication supports this hypothesis, there is a general perception that adverse events increased following the commercialization of the generic formulations. This lack of confidence in generics among medical oncologists may also be the consequence of several published clinical reports in which the generic formulation of other 
Table II. Hypersensitivity reactions (HSR) according to the type of oxaliplatin administered. Rash and itching were often associated.

\begin{tabular}{lccc}
\hline HSR & Total & Brand & Generic \\
\hline Total, n (\%) & $48(11.3)$ & $33(12.1)$ & $15(9.8)$ \\
Grade III-IV, n (\%) & $16(3.7)$ & $11(4.0)$ & $5(3.2)$ \\
Rash, n & 23 & 15 & 8 \\
Itching, n & 15 & 9 & 6 \\
Laryngospasm, n & 4 & 3 & 1 \\
Fever, n & 1 & 0 & 1 \\
Hypotension, n & 16 & 11 & 5 \\
\hline
\end{tabular}

drugs was more toxic (16-18), the concentration was lower than that declared (19), or the drug had lower efficacy than branded drugs $(20,21)$. However, it should be noted that several other studies did not find any difference between branded and generic drugs $(22,23)$. As far as oxaliplatin is concerned, only one study has reported a comparison between branded and generic formulations (24); in this retrospective study, a slight increase in non-hematological toxicities was documented in patients treated with generic formulation. However, the study included only a small number of patients, preventing any definitive conclusion.

Globally, the HSR incidence in our series was $11.3 \%$, whereas severe HSRs represented only $3.7 \%$ of the patients. These findings are in line with published data from the abovementioned review (22). In our study, we did not find any difference in HSR occurrence in patients treated with branded versus generic oxaliplatin formulation, no relevant difference in response rate and OS were demonstrated. Because of the equivalence of these two efficacy outcomes, we can explain the reported longer time to progression for the brand-treated group as being a consequence of differences in restaging procedures across the long period of time considered, or simply by chance. Additionally, our study was not planned and powered enough to detect such difference in time to progression.

The number of patients submitted to an oxaliplatin-based chemotherapy has grown in the past decade due to new indications, not only for patients with stage II/III CRC (25), but also for other malignancies such as pancreatic (26), biliary (27) and gastric (28) cancer. Having increased the number of patients at risk, it is reasonable to state that starting from the end of 2005, a larger and growing number of patients experienced an oxaliplatin-induced HSR. The new indications, especially those related to gastric cancer, appeared approximately at the same time when generic oxaliplatin entered the market (i.e. in 2008). Thus, a possible explanation about the current general feeling of an increase in oxaliplatin-induced HSRs with use of generics might simply rely on the association of these two events.
Table III. Maximal toxicities per patient in those submitted to FOLFOX (5-fluorouracil, leucovorin and oxaliplatin) XELOX (capecitabine and oxaliplatin) according to drug type.

\begin{tabular}{|c|c|c|c|c|}
\hline & Total & $\begin{array}{l}\text { Branded } \\
\text { drug }\end{array}$ & $\begin{array}{c}\text { Generic } \\
\text { drug }\end{array}$ & $\begin{array}{c}p \text {-Value* } \\
\text { (Branded } \\
v s . \\
\text { generic } \\
\text { drug) }\end{array}$ \\
\hline $\begin{array}{l}\text { No. of patients, } \mathrm{n} \\
\text { Leucopenia, n (\%) }\end{array}$ & \multicolumn{3}{|c|}{ Leucopenia, n (\%) } & \\
\hline Grade $0-1$ & $176(73.9)$ & $79(81.4)$ & $97(68.8)$ & \\
\hline Grade 2-4 & $62(26.1)$ & 18 (18.6) & $44(31.2)$ & 0.03 \\
\hline \multicolumn{5}{|l|}{ Neutropenia, n (\%) } \\
\hline Grade $0-1$ & $163(68.5)$ & $70(72.2)$ & $93(66.0)$ & \\
\hline Grade $2-4$ & $75(31.5)$ & $27(27.8)$ & $48(34.0)$ & 0.31 \\
\hline \multicolumn{5}{|c|}{ Thrombocytopenia, n (\%) } \\
\hline Grade $0-1$ & $204(85.7)$ & $81(83.5)$ & $123(87.2)$ & \\
\hline Grade 2-4 & $34(14.3)$ & $16(16.5)$ & $18(12.8)$ & 0.42 \\
\hline \multicolumn{5}{|l|}{ Anemia, n (\%) } \\
\hline Grade $0-1$ & $177(74.4)$ & $75(77.3)$ & $102(72.3)$ & \\
\hline Grade $2-4$ & $61(25.6)$ & $22(22.7)$ & $39(27.7)$ & 0.39 \\
\hline \multicolumn{5}{|l|}{ Nausea, n (\%) } \\
\hline Grade $0-1$ & $192(80.7)$ & $74(76.3)$ & $118(83.7)$ & \\
\hline Grade $2-4$ & $46(19.3)$ & $23(23.7)$ & $23(16.3)$ & 0.16 \\
\hline \multicolumn{5}{|l|}{ Diarrhea, n (\%) } \\
\hline Grade $0-1$ & $201(84.4)$ & $78(80.4)$ & $123(87.2)$ & \\
\hline Grade $2-4$ & $37(15.6)$ & $19(19.6)$ & $18(12.8)$ & 0.15 \\
\hline \multicolumn{5}{|l|}{ Mucositis, n (\%) } \\
\hline Grade $0-1$ & $222(93.3)$ & $88(90.7)$ & $134(95.0)$ & \\
\hline Grade $2-4$ & $16(6.7)$ & $9(9.3)$ & $7(5.0)$ & 0.19 \\
\hline \multicolumn{5}{|l|}{ Neuropathy, n (\%) } \\
\hline Grade $0-1$ & $204(85.7)$ & $82(84.5)$ & $122(86.5)$ & \\
\hline Grade 2 & $34(14.3)$ & $15(15.5)$ & $19(13.5)$ & 0.67 \\
\hline
\end{tabular}

*By $\chi^{2}$ test.

The reported toxicity burden in our series was mild, with a relatively low incidence of grade 3-4 side-effects. Thus, in order to increase the number of events per group, and to render toxicity comparison more sensible, we planned to stratify patients according whether they presented toxicity of grade 2 or more. No statistically significant difference in toxicity profiles between groups was evident $(p \geq 0.01)$.

We can conclude that the tested branded and generic oxaliplatin formulations had equivalent concentrations of the active agent, equivalent stability at different temperature conditions, and equivalent in vitro efficacy. The incidence of oxaliplatin-induced HSRs was not different according whether patients received branded or generic oxaliplatin. More relevantly, no difference in outcomes and toxicity profile was documented between the two groups.

Based on our data, it can be concluded that generic oxaliplatin should be considered as a safe and active alternative to the branded formulation. 


\section{References}

1 Shih YC, Ganz PA, Aberle D, Abernethy A, Bekelman J, Brawley O, Goodwin JS, Hu JC, Schrag D, Temel JS and Schnipper L: Delivering high-quality and affordable care throughout the cancer care continuum. J Clin Oncol 31(32): 4151-4157, 2013.

2 http://www.who.int/trade/glossary/story034/en/(accessed 03.01.2016).

3 Parel M, Ranchon F, Nosbaum A, You B, Vantard N, Schwiertz V, Gourc C, Gauthier N, Guedat MG, He S, Kiouris E, Alloux C, Vial T, Trillet-Lenoir V, Freyer G, Berard F and Rioufol C: Hypersensitivity to oxaliplatin: clinical features and risk factors. BMC Pharmacol Toxicol 15: 1, 2014.

4 Wang JH, King TM, Chang MC and Hsu CW: Oxaliplatininduced severe anaphylactic reactions in metastatic colorectal cancer: case series analysis. World J Gastroenterol 18(38): 54275433, 2012.

5 Ficarra R, Calabrò ML, Cutroneo P, Tommasini S, Melardi S, Semreen M, Furlanetto S, Ficarra P and Altavilla G: Validation of a LC method for the analysis of oxaliplatin in a pharmaceutical formulation using an experimental design. J Pharm Biomed Anal 29(6): 1097-2103, 2002.

6 http://www.atcc.org (accessed 03.01.2016)

7 Sprio AE, Di Scipio F, Ceppi P, Salamone P, Di Carlo F, Scagliotti GV, Papotti M, Ceccarelli A and Berta GN: Differentiation-inducing factor-1 enhances 5-fluorouracil action on oral cancer cells inhibiting E2F1 and thymidylate synthase mRNAs accumulation. Cancer Chemother Pharmacol 69(4): 983-989, 2012.

8 Common Terminology Criteria for Adverse Events, available from http://ctep.cancer.gov/protocolDevelopment/electronic_ applications/docs/ctcaev3.pdf (accessed 03.01.2016)

9 Giacchetti S, Bjarnason G, Garufi C, Genet D, Iacobelli S, Tampellini M, Smaaland R, Focan C, Coudert B, Humblet Y, Canon JL, Adenis A, Lo Re G, Carvalho C, Schueller J, Anciaux N, Lentz MA, Baron B, Gorlia T, Lévi F, European Organisation for Research and Treatment of Cancer Chronotherapy Group: Phase III trial comparing 4-day chronomodulated therapy versus 2-day conventional delivery of fluorouracil, leucovorin, and oxaliplatin as first-line chemotherapy of metastatic colorectal cancer: the European Organisation for Research and Treatment of Cancer Chronotherapy Group. J Clin Oncol 24(22): 35623569, 2006.

10 Miller AB, Hoogstroten B, Staquet M and Winkler A: Reporting results of cancer treatment. Cancer 47: 207-214, 1981.

11 Eisenhauer EA, Therasse P, Bogaerts J, Schwartz LH, Sargent D, Ford R, Dancey J, Arbuck S, Gwyther S, Mooney M, Rubinstein L, Shankar L, Dodd L, Kaplan R, Lacombe D and Verweij J: New response evaluation criteria in solid tumours: revised RECIST guideline (version 1.1). Eur J Cancer 45(2): 228-247, 2009.

12 http://www.ema.europa.eu/docs/en_GB/document_library/ Scientific_guideline/2010/01/WC500070039.pdf

13 Committee on Drugs: "Inactive" Ingredients in pharmaceutical products: Update (Subject Review). American Academy of Pediatrics Committee on Drugs. Pediatrics 99(2): 268-278, 1997.

$14 \mathrm{http} / / / \mathrm{www} . c p m e d i c a l . n e t / n e w s l e t t e r / t h e-p o t e n t i a l-h a z a r d s-o f-$ generic-drugs-medical (accessed 03.01.2016).

15 Toki MI, Saif MW and Syrigos KN: Hypersensitivity reactions associated with oxaliplatin and their clinical management. Expert Opin Drug Saf 13(11): 1545-1554, 2014.
16 Garrido-Siles M, Arenas-Villafranca JJ, Pérez-Ruiz E, de Linares Fernández MF, Tortajada B, Rivas-Ruiz F, Faus V and Rueda A: New cutaneous toxicities with generic docetaxel: are the excipients guilty? Support Care Cancer 23(7): 1917-1923, 2015.

17 Poirier E, Desbiens C, Poirier B, Hogue JC, Lemieux J, Doyle C, Leblond AF, Côté I, Cantin G and Provencher L: Comparison of serious adverse events between the original and a generic docetaxel in breast cancer patients. Ann Pharmacother 48(4): 447-455, 2014.

18 Oike T, Ohno T, Noda SE, Sato H, Tamaki T, Kiyohara H, Ando $\mathrm{K}$ and Nakano $\mathrm{T}$ : Comparison of hematological toxicities between innovator and generic cisplatin formulations in cervical cancer patients treated with concurrent chemoradiotherapy. J Radiat Res 54(3): 474-478, 2013.

19 Vial J, Cohen M, Sassiat P and Thiébaut D: Pharmaceutical quality of docetaxel generics versus originator drug product: a comparative analysis. Curr Med Res Opin 24(7): 2019-2033, 2008.

20 Madian AG, Panigrahi A, Perera MA and Pinto N: Case report: Inability to achieve a therapeutic dose of tacrolimus in a pediatric allogeneic stem cell transplant patient after generic substitution. BMC Pharmacol Toxicol 15: 69, 2014.

21 Mattar M: Failure of copy Imatib (CIPLA, India) to maintain hematologic and cytogenetic responses in chronic myeloid leukemia in chronic phase. Int J Hematol 91(1): 104-106, 2010.

22 Niho S, Yamanaka T, Umemura S, Matsumoto S, Yoh K, Goto $\mathrm{K}$, Ohmatsu $\mathrm{H}$ and Ohe $\mathrm{Y}$ : Renal toxicity caused by brand-name versus generic cisplatin: a comparative analysis. Jpn J Clin Oncol 43(4): 390-395, 2013.

23 De Lemos ML and Kyritsis V: Clinical efficacy of generic imatinib. J Oncol Pharm Pract 21(1):76-79, 2015.

24 Rasul KI, Kamal AS, Al-Najjar NM and Oculalan MH: Is there a difference between brand and generic oxaliplatin? Cancer Clin Oncol 1(2): 45-48, 2012.

25 André T, Boni C, Mounedji-Boudiaf L, Navarro M, Tabernero J, Hickish T, Topham C, Zaninelli M, Clingan P, Bridgewater J, Tabah-Fisch I and de Gramont A: Oxaliplatin, fluorouracil, and leucovorin as adjuvant treatment for colon cancer. N Engl J Med 350(23): 2343-2351, 2004.

26 Louvet C, Labianca R, Hammel P, Lledo G, Zampino MG, André T, Zaniboni A, Ducreux M, Aitini E, Taïeb J, Faroux R, Lepere C, de Gramont A; GERCOR, GISCAD: Gemcitabine in combination with oxaliplatin compared with gemcitabine alone in locally advanced or metastatic pancreatic cancer: results of a GERCOR and GISCAD phase III trial. J Clin Oncol 23(15): 3509-3516, 2005.

27 André T, Tournigand C, Rosmorduc O, Provent S, MaindraultGoebel F, Avenin D, Selle F, Paye F, Hannoun L, Houry S, Gayet B, Lotz JP, de Gramont A, Louvet C; GERCOR Group: Gemcitabine combined with oxaliplatin (GEMOX) in advanced biliary tract adenocarcinoma: a GERCOR study. Ann Oncol 15(9): 1339-1343, 2004.

28 Cunningham D, Starling N, Rao S, Iveson T, Nicolson M, Coxon F, Middleton G, Daniel F, Oates J and Norman AR: Capecitabine and oxaliplatin for advanced esophagogastric cancer. N Engl J Med 358(1): 36-46, 2008. 